\title{
Comparative study of ${ }^{99 \mathrm{~m}} \mathrm{Tc}-\mathrm{ECD}$ and ${ }^{99 \mathrm{~m}}$ Tc-HMPAO for peri-ictal SPECT: qualitative and quantitative analysis
}

Terence J O’Brien, Benjamin H Brinkmann, Brian P Mullan, Elson L So, Mary F Hauser, Michael K O'Connor, Joseph Hung, Clifford R Jack

\begin{abstract}
Objectives-Most studies that clinically validated peri-ictal SPECT in intractable partial epilepsy had used technetium99m-hexamethylpropylene amine oxime ( ${ }^{99 \mathrm{~m}}$ Tc-HMPAO or ${ }^{99 \mathrm{~m}}$ Tc-exametazime) as the radiopharmaceutical. Because of some theoretical advantages, technetium$99 \mathrm{~m}$-ethyl cysteinate diethylester $\left({ }^{99 \mathrm{~m}} \mathrm{Tc}-\right.$ ECD or ${ }^{99 \mathrm{~m}}$ Tc-bicisate) is increasingly being used instead. This study compares unstabilised ${ }^{{ }^{99} \mathrm{~m}}$ Tc-HMPAO and ${ }^{99 \mathrm{~m}} \mathbf{T c}-$ ECD in the performance of peri-ictal SPECT in partial epilepsy.
\end{abstract}

Methods-The injection timing and localisation rates in 49 consecutive patients with partial epilepsy who had peri-ictal injections with unstabilised ${ }^{99 \mathrm{~m}}$ Tc-HMPAO were compared with 49 consecutive patients who had peri-ictal injections with ${ }^{99 m}$ Tc-ECD. Quantitative cortical/subcortical and cortical/extracerebral uptake ratios were also compared. Subtraction SPECT coregistered to MRI (SISCOM) was performed in patients whose interictal SPECTS were available.

Results-In the ${ }^{99 \mathrm{~m}}$ Tc-ECD patients, the latency from seizure commencement to injection was shorter (median $34 v 80 \mathrm{sec}-$ onds, $\mathbf{p}<0.0001)$ and there was a lower rate of postictal injections $(16.3 \%$ v $57.1 \%$, $\mathbf{p}<0.0001)$. The cortical/extracerebral and cortical/subcortical uptake ratios were greater in the ${ }^{99 \mathrm{~m}} \mathrm{Tc}-\mathrm{ECD}$ images (median $5.0 v 3.6$, and $2.5 v 2.2$ respectively; both $p<0.005)$, but the relative peri-ictal increase in uptake in the cortical focus did not differ significantly (median $37.0 \% v$ $37.0 \% ; p>0.05)$. Blinded review of the SISCOM images were localising in a higher proportion of the ${ }^{99 \mathrm{~m}} \mathrm{Tc}-\mathrm{ECD}$ patients (40/45 (88.9\%) v 25/37 (67.6\%), p<0.05), and had a better concordance with EEG, MRI, and with the discharge diagnosis. Conclusion- ${ }^{99}$ Tc-ECD compares favourably with unstabilised ${ }^{99 \mathrm{~m}}$ Tc-HMPAO as a radiopharmaceutical for peri-ictal SPECT studies. Its use results in earlier injections and less frequent postictal injections than unstabilised ${ }^{99 \mathrm{~m}}$ Tc-HMPAO, thereby enhancing the sensitivity and the specificity of peri-ictal SPECT for the localisation of intractable partial epilepsy. (f Neurol Neurosurg Psychiatry 1999;66:331-339)

Keywords: Ictal SPECT; ${ }^{9{ }^{9 m}}$ Tc-ECD; ${ }^{99 \mathrm{~m}}$ Tc-HMPAO
Peri-ictal SPECT shows great promise as a tool to accurately localise seizures in intractable partial epilepsy, particularly in those with temporal lobe seizures. ${ }^{1-6}$ Moreover, the recent development of computer aided methods of subtracting the interictal SPECT images from the ictal images, with the subsequent coregistration of the difference images on to the MRI, has been shown to further improve the clinical usefulness of SPECT, especially in extratemporal epilepsy and in epilepsy that is difficult to localise. ${ }^{7-13}$ The radiopharmaceutical used in the vast majority of studies of peri-ictal SPECT has been technetium-99m-hexamethylpropylene amine oxime $\left({ }^{99 \mathrm{~m}} \mathrm{Tc}-\mathrm{HMPAO}\right.$ or ${ }^{99 \mathrm{~m}} \mathrm{Tc}-$ exametazime), which is unstable and needs to be reconstituted immediately before injection. ${ }^{14}$ Thus, the performance of true ictal SPECT studies with unstabilised ${ }^{99 \mathrm{~m}} \mathrm{Tc}-\mathrm{HMPAO}$ is difficult, particularly in patients with extratemporal seizures which are usually brief. Technetium-99m-ethyl cysteinate diethylester ( ${ }^{99 \mathrm{~m}}$ Tc-ECD or ${ }^{99 \mathrm{~m}}$ Tc-bicisate) is a relatively new brain SPECT perfusion radiopharmaceutical that has uptake kinetics and distribution similar to ${ }^{99 \mathrm{~m}} \mathrm{Tc}-\mathrm{HMPAO}$, but is stable in vitro for up to 6-8 hours after constitution. ${ }^{15}$ Therefore, it does not require mixing just before injection. As a result, the use of ${ }^{99 \mathrm{~m}}$ Tc-ECD may facilitate earlier injections, and thereby, may improve seizure localisation. ${ }^{16}{ }^{17}$

${ }^{99 \mathrm{~m}}$ Tc-ECD has only recently been used clinically for peri-ictal SPECT studies, with small preliminary studies reporting generally encouraging results. ${ }^{7816-19}$ However, some authors have suggested that the focal ictal uptake of ${ }^{99 \mathrm{~m}} \mathrm{Tc}-\mathrm{ECD}$ may not be as intense as with ${ }^{99 \mathrm{~m}}$ Tc-HMPAO. ${ }^{19-21}$ There is evidence that the two radiopharmaceuticals may show somewhat different distributions in some pathological conditions, ${ }^{22-26}$ which is likely related to different mechanisms of cerebral uptake..$^{23-31}$ Therefore, the sensitivity and the specificity of ${ }^{99 m}$ Tc-ECD need to be evaluated, especially in relation to those of the more established agent ${ }^{99 m}$ Tc-HMPAO. As yet, there has been no study that has directly compared these radiopharmaceuticals in peri-ictal studies of partial epileptic seizures.

In this study, we compared a group of consecutive intractable partial epilepsy patients who had peri-ictal SPECT using ${ }^{99 \mathrm{~m}} \mathrm{~T} \mathrm{c}-\mathrm{ECD}$ with another group of consecutive patients who had unstabilised ${ }^{99 m}$ Tc-HMPAO peri-ictal SPECT. The purpose was to determine whether the use of ${ }^{99 m}$ Tc-ECD was associated 
with shorter injection latencies and fewer postictal injections, and to compare its sensitivity and specificity for seizure localisation with those of ${ }^{99 \mathrm{~m}} \mathrm{Tc}-\mathrm{HMPAO}$. We also compared the radiopharmaceuticals for the magnitude of the ictal focal cortical increase in uptake, as well as for the cortical/subcortical and the cortical/ extracerebral uptake ratios.

\section{Methods}

PATIENT AND SPECT RADIOPHARMACEUTICAL SELECTION

We studied 49 consecutive patients with intractable partial epilepsy who had peri-ictal SPECT injections performed at the Mayo Medical Center, Rochester, MN, USA, using unstabilised ${ }^{99 \mathrm{~m}} \mathrm{Tc}-\mathrm{HMPAO}$ (June 1993January 1995) and another 49 consecutive patients in whom ${ }^{99 \mathrm{~m}} \mathrm{Tc}-\mathrm{ECD}$ was used (November 1993-January 1995). From November 1993 until January 1995, all adult patients being considered for peri-ictal SPECT study were offered the option of participating in an open label phase II trial using ${ }^{99 m}$ Tc-ECD for their SPECT study. (The trial was approved by our institutional review board.) During this period, 12 patients were studied with ${ }^{99 \mathrm{~m}} \mathrm{Tc}$ ECD and another 32 were studied with ${ }^{99 \mathrm{~m}} \mathrm{Tc}$ HMPAO. After this period, ${ }^{99 \mathrm{~m}}$ Tc-ECD was used for all peri-ictal SPECT.

CLINICAL AND SEIZURE INFORMATION

The patents' medical records were abstracted for demographic and clinical information. Video tapes of the injected seizures were reviewed for the time of onset and termination of clinical seizures, and the timing of the injection. Seizure onset was defined as the time of earliest indication of a warning (verbalised or pushing the call button), or of abnormal movements, behaviour, or impaired awareness. The end of a seizure was the time when the ictal movements or behaviour ceased. When the start and/or the end of the seizure could not be confidently established from clinical features, the beginning and/or the end of the rhythmic seizure discharge on the ictal EEG was used for the seizure timing. The injections were divided into those that were "ictal" (the injection was performed while there was continuing clinical and/or electrographic seizure activity), and those that were "postictal".

RADIOPHARMACEUTICAL PREPARATION AND INJECTION METHODS

${ }^{99 \mathrm{~m}}$ Tc-HMPAO was prepared in accordance with the product information guidelines using the Ceretec kit (Amersham Corporation, Arlington Heights, Illinois, IL, USA) ${ }^{14}$ In the Nuclear Medicine radiopharmaceutical laboratory, approximately $100 \mathrm{mCi}(3.7 \mathrm{GBq})$ of sterile freshly eluated, oxidant free ${ }^{99 m} \mathrm{Tc}$ sodium pertechnetate was diluted up to $5 \mathrm{ml}$ with $0.9 \%$ sodium chloride in a shielded syringe. This was then placed in a lead lined container and transported to the epilepsy monitoring unit for storage until use. At the time the patient's seizure was noted to commence, the EEG technician injected the contents of the ${ }^{99 \mathrm{~m}} \mathrm{Tc}$ sodium pertechnetate syringe into the Ceretec vial. After the solution was thoroughly mixed for about 10 seconds, the appropriate dose for injection was then withdrawn from the vial for injection into the patient. This procedure of radiopharmaceutical preparation took a trained technician 30-40 seconds to complete.

${ }^{99 \mathrm{~m}}$ Tc-ECD was labelled and reconstituted using the Neurolite kit (Du Pont Merck Pharmaceutical Company, Billerica, MA, USA) in accordance with the product information, ${ }^{15}$ before delivery to the epilepsy monitoring unit. $100 \mathrm{mCi}\left(3.7 \mathrm{GBq}\right.$ ) sterile, oxidant free ${ }^{99 \mathrm{~m}} \mathrm{Tc}$ sodium pertechnetate in approximately $2.0 \mathrm{ml}$ was added to the buffer vial (vial B). The lyophilised ligand vial (vial A) was reconstituted by the addition of $3.0 \mathrm{ml} 0.9 \%$ sodium chloride, and then $1.0 \mathrm{ml}$ of this solution was added to vial $\mathrm{B}$. The preparation was thoroughly mixed for a few seconds and allowed to stand for 30 minutes. ${ }^{15}$ A rapid preparation technique was alternatively used in which the contents were microwaved for 8 seconds at 300 W. ${ }^{32}$ The radiochemical purity was checked using paper chromatography to ensure that it was greater than $90 \% .^{33}$ The solution was then drawn into a shielded syringe, placed into a lead lined container, and transported to the epilepsy monitoring unit.

The peri-ictal radiopharmaceutical injections were performed by epilepsy technicians during a typical seizure while the patients were undergoing video-EEG monitoring in our inpatient epilepsy monitoring unit. The technicians had been specifically trained to perform the injections, which included taking a course in radiation safety. The interictal injections were performed when the patient had been seizure free for at least 24 hours, in standard ambient room lighting with eyes open and ears unplugged. The radiopharmaceutical dose injected was approximately $20 \mathrm{mCi}(740 \mathrm{MBg}$ ) for all studies.

\section{SPECT METHODS}

Using an identical protocol for both studies, the SPECT images were acquired with the same scanner within 2 to 3 hours of the radiopharmaceutical injection. A dual head gamma camera system (Helix, Elscint Inc, Haifa, Israel) equipped with ultrahigh resolution fan beam collimators was employed. The data were acquired in a $128 \times 128$ byte matrix over 360 degrees, with 120 views obtained at 3 degree intervals for 15 seconds per view using a circular orbit. The energy setting was 140 $\mathrm{keV}$ with a $15 \%-20 \%$ window. Transaxial images were reconstructed using a Metz filter (power=3, full width at half maximum $(\mathrm{FWHM})=6 \mathrm{~mm})$ rebinned into a $64 \times 64$ matrix with a $2 \times$ zoom. Attenuation correction was applied and images were viewed in the usual three orthogonal planes. The reconstructed system resolution was approximately 7 mm FWHM, consisting of cubic voxels with dimensions of 3.6 or $4.4 \mathrm{~mm}$ (depending on the image size). Images were reconstructed using a standard filtered back projection algorithm in the coronal, sagittal, transaxial, and transtemporal planes. 
For all patients in whom both an interictal SPECT scan and a brain MRI were available (37 of the ${ }^{99 \mathrm{~m}} \mathrm{Tc}-\mathrm{HMPAO}$ patients and 45 of the ${ }^{99 \mathrm{~m}}$ Tc-ECD patients) subtraction ictal SPECT coregistered to MRI (SISCOM) images (fig 1) were constructed on an off line Unix based workstation with the aid of commercial image analysis software packages ${ }^{7}$ (ANALYZE 7.5 and Analyze/AVW, Biomedical Imaging Resource, Mayo Foundation, Rochester, MN, USA) and with the use of a chamfer distance based surface matching technique $^{34}$.

\section{QUANTITATIVE SPECT ANALYSIS}

The quantitative SPECT studies were performed by a single operator (BHB) who was blinded to clinical details, results of other investigations, and results of the qualitative analysis. For the quantitative analysis, SPECT images were reformatted so that they were all composed of cubic voxels with the same dimensions $(1.8 \times 1.8 \times 1.8 \mathrm{~mm})$. Six circular regions of interest (ROIs) with a $9.0 \mathrm{~mm}$ diameter were used to measure the relative uptake in the neocortex, the subcortical white matter, and the extracranial tissue. On a single coronal slice at the mid-temporal level, these ROIs were respectively placed bilaterally over the parietal cortex, the adjacent parietal subcortical white matter, and extracerebral tissue, as shown in fig $2 \mathrm{~A}$, for both the ictal and the interictal studies. The mean intensity within each of these ROIs was measured, and the corresponding right and left ROI values for each region were averaged to minimise the influence of the side of lateralisation of the epileptogenic zone. The cortical/subcortical and the cortical/extracerebral uptake ratios were then calculated for both the ictal and interictal studies to minimise the effects of any focal cortical hyperperfusion in the ictal studies or focal hypoperfusion in the interictal studies. These results were compared between the ${ }^{99 \mathrm{~m}} \mathrm{Tc}$ HMPAO patients and the ${ }^{99 \mathrm{~m}} \mathrm{Tc}-\mathrm{ECD}$ patients. The rationale for comparing these ratios was to determine whether ${ }^{99 \mathrm{~m}} \mathrm{Tc}-\mathrm{ECD}$ has a superior brain to extracerebral or cortical to white matter contrast, which has been stated as a potential advantage of the agent.
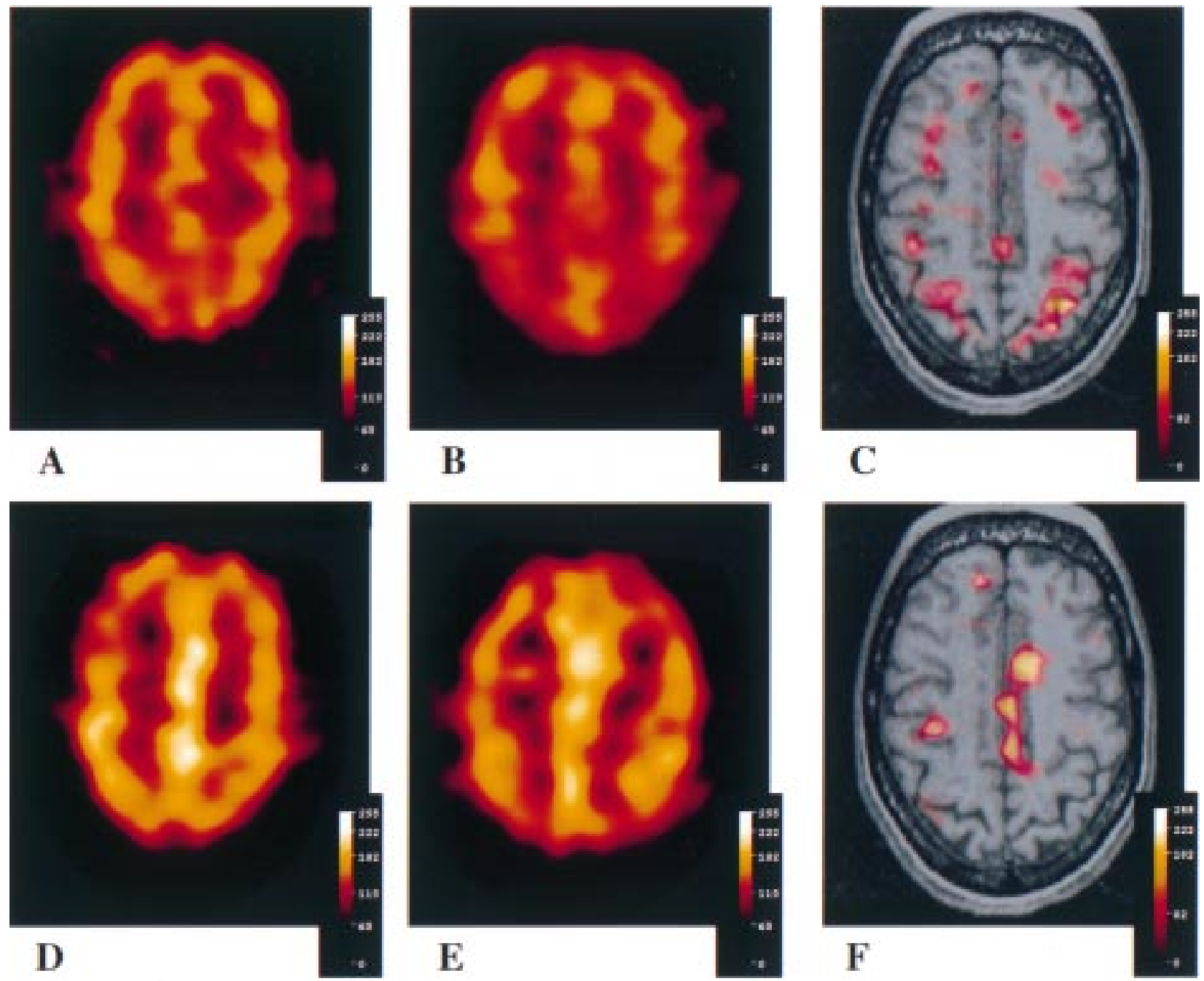

Figure 1 SPECT images of a patient with intractable non-lesional extratemporal seizures. The initial SPECT studies were done with ${ }^{99 m} T c-H M P A O:$ $((A)$ postictal image, $(B)$ interictal image, and $(C)$ SISCOM). The injection was postictal, resulting in a non-localising SISCOM image. A repeat ictal study and an interictal study were performed using ${ }^{99_{m}} T c-E C D$ ( D) ictal image, (E) interictal image, and (F) SISCOM). The ictal injection of

${ }_{99 m} T c-E C D$ resulted in a localised SISCOM abnormality in the left mesial frontal lobe, which was concordant with seizure semiology and with ictal EEG localisation. 

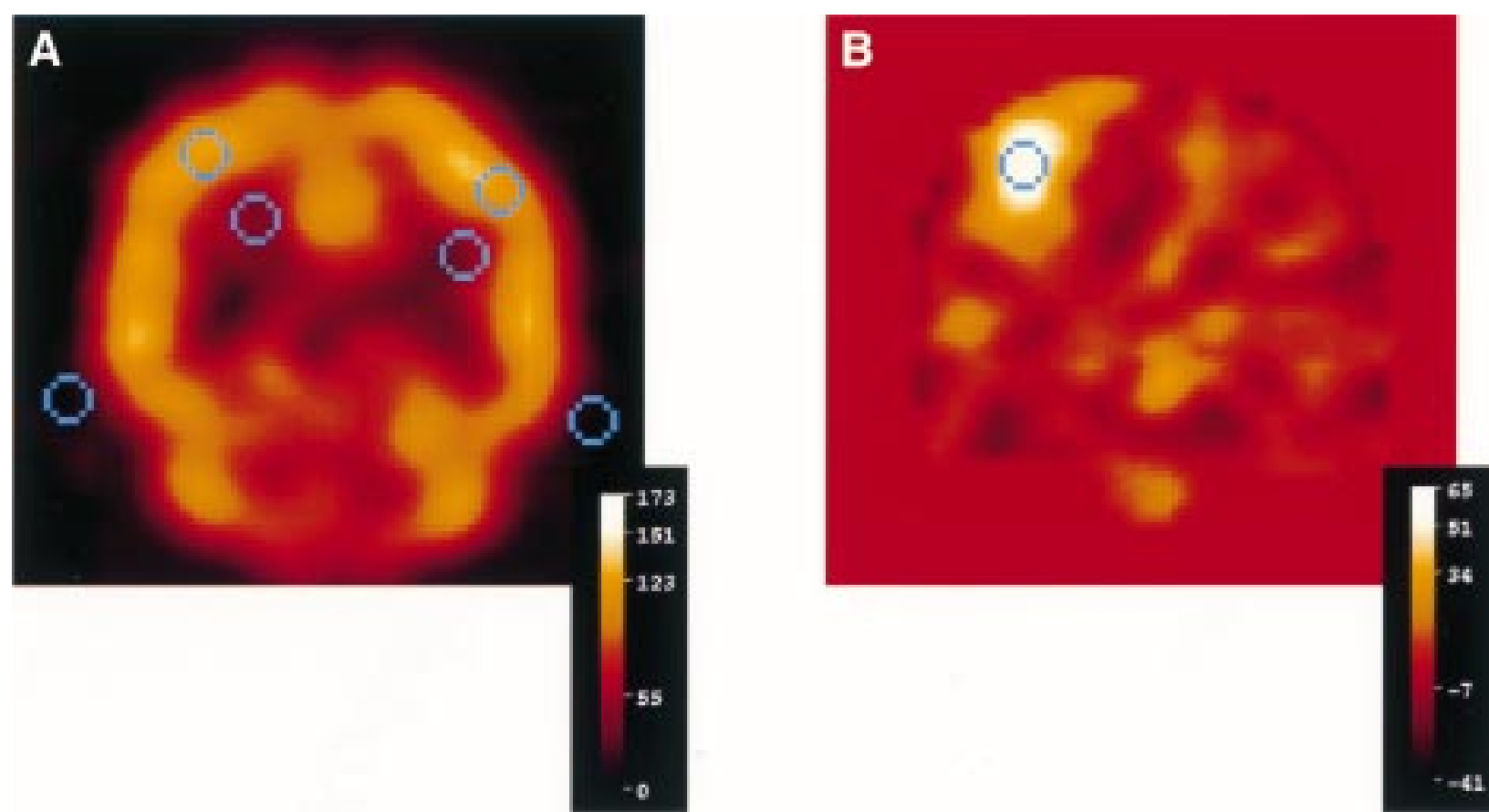

Figure 2 (A) Coronal SPECT image from a ${ }^{99 m}$ Tc-HMPAO injection showing the sites of placement of the 9 mm diameter circular ROIs for the quantitative measurement of the cortical/subcortical and corticallextracerebral uptake ratios. (B) Coronal non-thresholded subtraction SPECT image from a patient injected with ${ }^{99 m} \mathrm{Tc}-E C D$, showing the site of placement of the $9 \mathrm{~mm}$ ROI for quantification of the percentage ictal increase in uptake at the cortical focus.

To quantify the magnitude of the maximal focal cortical ictal increase in uptake, a single circular ROI was placed over the visually identified focus of hyperperfusion on the unthresholded subtraction image (fig $2 \mathrm{~B}$ ). The mean intensity value for this ROI was then calculated and expressed as a percentage increase in intensity from the interictal to the ictal image. This value was then compared between the ${ }^{99 \mathrm{~m}} \mathrm{Tc}-\mathrm{HMPAO}$ and the ${ }^{99 \mathrm{~m}} \mathrm{Tc}-\mathrm{ECD}$ patients.

BLINDED QUALITATIVE SPECT INTERPRETATION Both the traditional visual side by side ictal and interictal scans and the SISCOM images were interpreted independently by two reviewers (BPM and ELS) who were blinded to the clinical data, the SPECT radiopharmaceutical group, and the results of other tests. The reviewers were told whether the injections were ictal or postictal, and if postictal, they were also informed of the seizure length and injection timing. For the traditional visual interpretation, both the colour scale and grey scale images were used. For the SISCOM review, only 2SD images were used for those with ictal injection, but both the 1SD and 2SD images were used for those with postictal injections. The reviewers were asked to localise the images to one of 16 sites (either right or left: frontal, frontotemporal, temporal, frontoparietal temporoparietal, parietal, occipitoparietal, or occipital), or classify them as being nonlocalising. Final determination was based on the agreement of the reviewers on the localisation or non-localisation of the studies. If the two reviewers disagreed, a third blinded reviewer was used $(\mathrm{MFH})$. Agreement of the third reviewer with one of the primary reviewers served as the final determination. If the third reviewer's localisation did not agree with one of the primary reviewers, the study was considered non-localising.

COMPARISON WITH THE EEG AND MRI LOCALISATION AND DISCHARGE DIAGNOSIS. Brain MRI was performed according to a standardised seizure protocol ${ }^{35}$ on a 1.5 Tesla Signa scanner (GE Medical Systems, Milwaukee, WI, USA). Ictal scalp-EEG was recorded using a 32 channel system with the electrodes arranged according to a modified 10-20 system that included subtemporal electrodes. Thirty of the patients had further prolonged monitoring with intracranial electrodes (14 of the ${ }^{99 \mathrm{~m}} \mathrm{Tc}-$ HMPAO patients and 16 of the ${ }^{99 \mathrm{~m}} \mathrm{Tc}-\mathrm{ECD}$ patients). Thirteen had subdural strips/grids, four had bitemporal depth electrodes, and 13 had a combination of both strip/grids and depth electrodes. The localisations by ictal scalp EEG, ictal intracranial EEG, and seizure protocol MRI were determined by retrospective review of their reports. The discharge diagnosis was that given by the epileptologist on completion of the video-EEG monitoring, and was based on an assimilation of all localising or lateralising information available, except that of the SPECT studies (for example, the results of MRI, prolonged video-EEG, and the clinical history). The discharge diagnosis, as well as the localisations by ictal scalp EEG, ictal intracranial EEG, and MRI, were compared with the localisation by traditional review of SPECT images and with the localisation by SISCOM images.

\section{STATISTICAL ANALYSIS}

Two tailed Fisher's exact test was used for comparisons between proportions. Student's $t$ test (two tailed) was applied for comparisons of continuous variables that were approximately 
Table 1 Comparison of patients who had ${ }^{99 m}$ Tc-HMPAO SPECT with those who had ${ }^{99 m}$ Tc-ECD SPECT according to their demographic data, discharge diagnoses, seizure length, and injection timing

\begin{tabular}{llll}
\hline & $\begin{array}{l}99 m T c-H M P A O \\
(n=49)\end{array}$ & $\begin{array}{l}99 m T c-E C D \\
(n=49)\end{array}$ & $p$ Value \\
\hline Age (y); median (range) & $26(1.5-69)$ & $30(6-54)$ & NS \\
Male/female & $25 / 24$ & $27 / 22$ & NS \\
Discharge diagnosis; temporal/others & $17 / 32$ & $21 / 28$ & NS \\
Seizure length (seconds); median (range) & $70(5-314)$ & $55(19-268)$ & NS \\
Injection latency (seconds); median (range) & $80(3-260)$ & $34(6-182)$ & $<0.0001$ \\
Ictal/postictal injections & $21 / 28$ & $41 / 8$ & $<0.001$ \\
\hline
\end{tabular}

${ }^{\text {a Others }}=$ extratemporal or unlocalised partial epilepsy.

Table 2 Discharge diagnoses in the 98 consecutive patients with intractable partial epilepsy who had peri-ictal SPECTs performed

\begin{tabular}{llll}
\hline \multicolumn{4}{c}{ Numbers of patients } \\
\cline { 2 - 4 } Discharge diagnosis & $\begin{array}{l}\text { 99mTc-HMPAO } \\
(n=49)\end{array}$ & $\begin{array}{l}99 m T c-E C D \\
(n=49)\end{array}$ & $\begin{array}{l}\text { All injections } \\
(n=98)\end{array}$ \\
\hline Temporal lobe epilepsy & $17(34.7 \%)$ & $21(42.9 \%)$ & $38(38.8 \%)$ \\
Extratemporal epilepsy & $22(44.9 \%)$ & $22(44.9 \%)$ & $44(44.9 \%)$ \\
$\quad$ Frontal & 10 & 10 & 20 \\
Frontotemporal & 4 & 2 & 6 \\
Frontoparietal & 3 & 3 & 6 \\
Temproparietal & - & 1 & 6 \\
Parietal & 3 & 1 & 1 \\
Occipitoparietal & 1 & 2 & 1 \\
Occipitotemporal & 1 & $6(12.2 \%)$ & $16(16.3 \%)$ \\
Occipital & $10(20.4 \%)$ & 3 \\
Unlocalised & & & 1 \\
\hline
\end{tabular}

Discharge diagnosis=diagnosis made by the epileptologist on discharge from the epilepsy monitoring unit. tests). ${ }^{99 \mathrm{~m}}$ Tc-ECD patients also had a significantly lower proportion of postictal injections than ${ }^{99 m}$ Tc-HMPAO patients $(16.3 \%$ v $57.1 \%$; $\mathrm{p}<0.001$, Fisher's exact test). The duration of the injected seizure did not significantly differ between the groups. When the analysis was restricted to patients who had peri-ictal SPECT during the time period when both ${ }^{99 \mathrm{~m}}$ Tc-HMPAO and ${ }^{99 \mathrm{~m}}$ Tc-ECD were being used, ${ }^{99 m}$ Tc-ECD patients $(n=12)$ again had a significantly shorter injection latency than ${ }^{99 m}$ Tc-HMPAO patients $(\mathrm{n}=32)$ (median 41 , range 19-58 seconds, $v$ median 77 , range 3-194 seconds; $\mathrm{p}<0.01$, Student's $t$ test) and a lower proportion of postictal injections $(8.3 \% v$ $56.3 \%$; $<<0.01$, Fisher's exact test). The duration of the injected seizures again did not significantly differ between the groups (median 56.5 , range $22-172, v$ median 61.5 , range 5-314 seconds; $\mathrm{p}>0.05$, Student's $t$ test).

QUANTITATIVE SPECT ANALYSIS

The median value for the cortical/ extracerebral ROI intensity ratios was significantly greater for the ECD images than the ${ }^{99 \mathrm{~m}}$ Tc-HMPAO images (median 5.0 v 3.6; $\mathrm{p}<0.0 .001$, Mann-Whitney $U$ test). Median value for the cortical/subcortical ROI intensity ratios was also greater for the ${ }^{99 \mathrm{~m}} \mathrm{Tc}-\mathrm{ECD}$ images (2.5 $v 2.2 ; \mathrm{p}<0.01$, Mann-Whitney $U$ test).

There was no difference in the relative ictal increase in uptake in the cortical focus between the subtraction images of ${ }^{99 \mathrm{~m}} \mathrm{Tc}-\mathrm{ECD}$ and of ${ }^{99 m}$ Tc-HMPAO studies (median $37.0 \%$ v $37.0 \%$; p >0.05, Mann-Whitney $U$ test). Also, there was no significant difference between the radiopharmaceutical groups when subgroup analyses were performed on patients with ictal injections $(37.3 \% v 34.1 \%, \mathrm{p}>0.05)$ or on patients with postictal injections $(34.6 \% v$ $40.8 \%, \mathrm{p}>0.05)$.

\section{Results}

DEMOGRAPHIC, CLINICAL, AND INJECTION DETAILS Of the 98 patients in the study, $52(53.1 \%)$ were male and $46(46.9 \%)$ were female. The median age of the patients was 28 years with a range of 1.5-69 years. Table 1 compares the demographic details, discharge diagnoses, and seizure and injection timing between patients who had ${ }^{99 \mathrm{~m}} \mathrm{Tc}-\mathrm{HMPAO}$ and patients who had ${ }^{99 \mathrm{~m}} \mathrm{Tc}-$ ECD injections. There was no significant difference between the two groups in age, sex, and proportion of patients with temporal versus extratemporal or unlocalised partial epilepsy (all $\mathrm{p}>0.05$ ). The details of the discharge diagnoses in the two groups are given in table 2.

${ }^{99 m}$ Tc-ECD patients had a significantly shorter injection latency $(\mathrm{p}<0.001$, Student's $t$
BLINDED SPECT INTERPRETATION

Fifteen patients did not have an interictal SPECT performed and one patient did not have review of the SISCOM images and the traditional side by side ictal-interictal SPECT images was performed on the remaining patients (37 in the ${ }^{99 \mathrm{~m}} \mathrm{Tc}-\mathrm{HMPAO}$ group and 45 in the ${ }^{99 \mathrm{~m}}$ Tc-ECD group). The results of the blinded analysis for the SISCOM images are summarised in table 3. Overall, ${ }^{99 \mathrm{~m}} \mathrm{Tc}-\mathrm{ECD}$ studies were determined to be localising in a significantly higher proportion than ${ }^{99 \mathrm{~m}} \mathrm{Tc}-\mathrm{HMPAO}$ studies $(\mathrm{p}<0.05$, Fisher's exact test). However, when patients who had ictal injections were analysed MRI available for coregistration. The blinded

Table 3 Comparison of the results of blinded analysis of SISCOM images

\begin{tabular}{|c|c|c|c|c|c|c|}
\hline & \multicolumn{2}{|l|}{ Ictal injections } & \multicolumn{2}{|c|}{ Postictal injections } & \multicolumn{2}{|l|}{ All injections } \\
\hline & $\begin{array}{l}{ }^{99 m} T c-H M P A O \\
(n=13)\end{array}$ & $\begin{array}{l}{ }^{99 m} T c-E C D \\
(n=37)\end{array}$ & $\begin{array}{l}{ }^{99 m} T c-H M P A O \\
(n=24)\end{array}$ & $\begin{array}{l}{ }^{99_{m}} T c-E C D \\
(n=8)\end{array}$ & $\begin{array}{l}{ }^{99 m} T c-H M P A O \\
(n=37)\end{array}$ & $\begin{array}{l}{ }^{99 m} T c-E C D \\
(n=45)\end{array}$ \\
\hline Localising & $11(84.6 \%)$ & $33(89.2 \%)$ & $14(58.3 \%)$ & $7(87.5 \%)$ & $25(67.6 \%)^{\star}$ & $40(88.9 \%)^{\star}$ \\
\hline Temporal & 3 & 11 & 6 & 4 & 9 & 15 \\
\hline Extratemporal & 8 & 22 & 8 & 3 & 16 & 25 \\
\hline Interobserver agreement & $76.9 \%$ & $86.5 \%$ & $58.3 \%$ & $62.5 \%$ & $64.9 \%$ & $82.2 \%$ \\
\hline Kappa score & 0.74 & 0.85 & 0.53 & 0.55 & $0.62^{\star}$ & $0.80^{\star}$ \\
\hline
\end{tabular}

${ }^{\star} \mathrm{p}<0.05 ;{ }^{99 \mathrm{~m}} \mathrm{Tc}-\mathrm{HMPAO} v^{99 \mathrm{~m}} \mathrm{Tc}$-ECD groups. 
Table 4 Concordance of the blinded SISCOM localisation with that of MRI, ictal scalp EEG, ictal intracranial EEG, or the final discharge diagnosis when both were localising

\begin{tabular}{|c|c|c|c|c|c|c|}
\hline & \multicolumn{2}{|l|}{ Ictal injections } & \multicolumn{2}{|c|}{ Postictal injections } & \multicolumn{2}{|l|}{ All injections } \\
\hline & $\begin{array}{l}{ }^{99 m} T c-H M P A O \\
(n=13)\end{array}$ & $\begin{array}{l}{ }^{99 m} T c-E C D \\
(n=37)\end{array}$ & $\begin{array}{l}{ }^{99 m} T c-H M P A O \\
(n=24)\end{array}$ & $\begin{array}{l}{ }^{99 m} T c-E C D \\
(n=8)\end{array}$ & $\begin{array}{l}{ }^{99 m} T c-H M P A O \\
(n=37)\end{array}$ & $\begin{array}{l}{ }^{99 m} T c-E C D \\
(n=45)\end{array}$ \\
\hline MRI & $4 / 5(80.0 \%)$ & $15 / 16(93.8 \%)$ & $5 / 8(62.5 \%)$ & $2 / 3(66.7 \%)$ & $9 / 13(69.2 \%)$ & $17 / 19(89.5 \%)$ \\
\hline Ictal Scalp EEG & $5 / 5(100 \%)$ & $23 / 26(88.5 \%)$ & $6 / 12(50.0 \%)$ & $3 / 4(75 \%)$ & $11 / 17(64.7 \%)$ & $26 / 30(86.7 \%)$ \\
\hline Ictal Intracranial EEG & $3 / 3(100 \%)$ & $10 / 10(100 \%)$ & $0 / 5(0 \%)$ & $1 / 2(50 \%)$ & $3 / 8(37.5 \%)^{\star}$ & $11 / 12(91.7 \%)^{\star}$ \\
\hline Epilepsy diagnosis & $8 / 9(88.9 \%)$ & $24 / 29(82.8 \%)$ & $4 / 13(30.8 \%)$ & $4 / 6(66.7 \%)$ & $12 / 22(54.5 \%)$ & $28 / 35(80.0 \%)$ \\
\hline
\end{tabular}

separately from those with postictal injections, there was no significant difference in the localisation rates between the radiopharmaceutical groups. The interobserver agreement between the two primary reviewers was significantly higher for the ${ }^{99 \mathrm{~m}} \mathrm{Tc}-\mathrm{ECD}$ images than for the ${ }^{99 \mathrm{~m}} \mathrm{Tc}-\mathrm{HMPAO}$ images $(\kappa=0.80$ v $\kappa=0.61$, $\mathrm{p}<0.05)$. However, the interobserver agreement was not significantly different between the radiopharmaceutical groups when those with ictal injections were analysed separately from those with postictal injections.

Using the traditional side by side visual comparison of the SPECT, there was no significant difference between ${ }^{99 \mathrm{~m}} \mathrm{Tc}-\mathrm{ECD}$ and ${ }^{99 m} \mathrm{Tc}-\mathrm{HMPAO}$ studies in the proportion that were localising $(32.4 \%$ v $37.8 \%$; $>>0.05$, Fisher's exact test), but the interobserver agreement was significantly better for the ${ }^{99 \mathrm{~m}} \mathrm{Tc}$ ECD studies $(51.1 \%$ v $37.8 \%$; $\kappa=0.43 v 0.29$, $\mathrm{p}<0.05)$. Compared with side by side visual comparison of SPECT images, the SISCOM method was localising in a significantly higher proportion of both ${ }^{99 \mathrm{~m}} \mathrm{Tc}-\mathrm{ECD}$ and ${ }^{99 \mathrm{~m}} \mathrm{Tc}$ HMPAO patients (both $\mathrm{p}<0.005$, Fisher's exact test), and for either ictal (88.0\% v 38.0\%, $\mathrm{p}<0.0001)$ or postictal injection $(65.6 \% v$ $40.0 \%, \mathrm{p}<0.005)$.

COMPARISON WITH MRI, EEG LOCALISATION, AND DISCHARGE DIAGNOSIS

The seizure protocol MRI showed a potentially epileptogenic focal lesion in 50 patients $(24 / 49$ of the ${ }^{99 \mathrm{~m}} \mathrm{Tc}-\mathrm{HMPAO}$ patients and 26/49 of the ${ }^{99 m}$ Tc-ECD patients, $\left.p>0.05\right)$. The types of lesions were: mesial temporal sclerosis 21, encephalomalacia 12, focal cortical dysgenesis 10 , low grade tumour six, cavernoma one. The ictal scalp EEG was localising in 67 patients (33 of the ${ }^{99 \mathrm{~m}} \mathrm{Tc}-\mathrm{HMPAO}$ and 34 of the ${ }^{99 \mathrm{~m}} \mathrm{Tc}-$ ECD patients, $p>0.05)$. Intracranial recordings were localising in $27 / 30(90 \%)$ patients $(14 / 14$ of the ${ }^{99 m}$ Tc-HMPAO and $13 / 16$ of the ${ }^{99 \mathrm{~m}}$ Tc-ECD patients, $\mathrm{p}>0.05$ ).

Table 4 summarises the concordance of the SISCOM localisation with the MRI, the EEG localisations, or the discharge diagnosis in the patients in whom both were localising or lateralising. Compared with ${ }^{99 \mathrm{~m}} \mathrm{Tc}-\mathrm{HMPAO}$, a significantly higher proportion of the ${ }^{99 \mathrm{~m}} \mathrm{Tc}$ ECD localisations agreed with the intracranial EEG localisation ( $p<0.05$, Fisher's exact test). Compared with ${ }^{99 m} \mathrm{Tc}-\mathrm{HMPAO}$, there was also a trend for a greater proportion of ${ }^{99 \mathrm{~m}} \mathrm{Tc}-\mathrm{ECD}$ localisations to be concordant with the discharge diagnosis $(p=0.07)$ and with the scalp ictal EEG localisation $(p=0.11)$. However, there was no significant difference between radiopharmaceutical groups when the subgroups of ictal and postictal injections were analysed separately. No significant difference was found between ${ }^{99 \mathrm{~m}} \mathrm{Tc}-\mathrm{HMPAO}$ and ${ }^{99 \mathrm{~m}} \mathrm{Tc}-$ ECD groups in the concordance of localisation by the traditional SPECT review with the localisation by each of the following: MRI (7/9 $(77.8 \%)$ v 10/12 (83.3\%), p>0.05), ictal scalp EEG (7/9 (77.8\%) v 12/13 (92.3\%), p>0.05), ictal intracranial EEG $(2 / 2(100 \%)$ v $4 / 5$ $(80 \%), \mathrm{p}>0.05)$, discharge diagnosis $(7 / 10$ $(70.0 \%) v 14 / 15(93.3 \%), \mathrm{p}>0.05$.

\section{Discussion}

To our knowledge, this is the first study to compare the use of ${ }^{99 m}$ Tc-ECD with that of ${ }^{99 m}$ Tc-HMPAO in peri-ictal SPECT. We found that the use of ${ }^{99 \mathrm{~m}}$ Tc-ECD is associated with significantly earlier SPECT injections than using unstabilised ${ }^{99 \mathrm{~m}} \mathrm{Tc}$-HMPAO, resulting in a significantly increased rate of obtaining an ictal SPECT study. These findings are not surprising and almost certainly are a direct result of not having to reconstitute the ${ }^{99 \mathrm{~m}} \mathrm{Tc}-\mathrm{ECD}$ just before injection, which we found took 30-40 seconds for the unstabilised ${ }^{99 \mathrm{~m}} \mathrm{Tc}-$ HMPAO to be prepared. Because many of our ${ }^{99 m}$ Tc-ECD studies were performed more recently than our ${ }^{99 \mathrm{~m}} \mathrm{Tc}-\mathrm{HMPAO}$ studies, it is possible that improvement in technician skills over time was partly responsible for the earlier injection timing with ${ }^{99 \mathrm{~m}} \mathrm{Tc}-\mathrm{ECD}$. However, when analysis was restricted to patients who were injected during the time period when both radiopharmaceuticals were being used, the ${ }^{99 \mathrm{~m}}$ Tc-ECD patients still had a significantly shorter injection latency and a higher proportion of ictal injections. We also do not think that our results can be explained by differences in ${ }^{99 \mathrm{~m}} \mathrm{Tc}-\mathrm{ECD}$ and ${ }^{99 \mathrm{~m}} \mathrm{Tc}-\mathrm{HMPAO}$ patient populations, as the two groups were well matched in the duration of injected seizures and in the proportions of each of following features: extratemporal epilepsies, MRI focal lesions, localising scalp EEG, and need for intracranial EEG recording (tables 1 and 2).

A stabilised formulation of ${ }^{99 \mathrm{~m}} \mathrm{Tc}-\mathrm{HMPAO}$ that does not require reconstitution immediately before injection has recently been approved for use in the United States. However, this is still not available or widely used in many countries. One of the arguments that has been put forward against the introduction of the more expensive stabilised radiopharmaceuticals is that shorter injection latency has not been previously proved to result in clinically significant improvement of seizure localisation. 
The results of our study also show that the use of ${ }^{99 \mathrm{~m}} \mathrm{Tc}-\mathrm{ECD}$ is associated with a significantly higher proportion of localising SISCOM studies than unstabilised ${ }^{99 \mathrm{~m}}$ Tc-HMPAO. The most important factor for this improvement in the sensitivity seems to be the shorter injection latency and the resultant fewer numbers of postictal injections. When patients with ictal and with postictal injections were analysed separately, there was no significant difference between the two radiopharmaceuticals in the rates of localisation. Also, the significantly lower interobserver agreement for the ${ }^{99 \mathrm{~m}} \mathrm{Tc}$ HMPAO studies for both the SISCOM and the traditional visual analysis methods seems to be primarily explained by the difference in injection time; the difference between the two radiopharmaceuticals was not significant when the ictal injections and the postictal injections were analysed separately.

The blinded SISCOM analysis had a significantly higher localisation rate than the traditional side by side review of SPECT images for both radiopharmaceutical groups, and for both ictal and postictal injections. In a previous study, we had shown that SISCOM had a superior localisation rate with ictal studies. ${ }^{8}$ In this study, we extend this finding to postictal studies. In both studies, the sensitivity of the traditional method of reviewing peri-ictal SPECT images has been somewhat less than previously reported. ${ }^{2638-43}$ The difference could be explained by our patient population, almost two thirds of which had extratemporal or unlocalised seizures, and almost half had non-lesional epilepsies. By contrast, almost all previous studies of peri-ictal SPECT were of selected groups of patients with mostly temporal lobe epilepsy or with seizures that were already well localised by other means. ${ }^{2}{ }^{639-48}$ Moreover, our protocol for the blinded review of the SPECT studies was more exacting than most previous studies, which have generally required the reviewers to select from only a limited number of possible localisations, or to just lateralise the SPECT abnormality. ${ }^{2}{ }^{6}$ 39-48

Overall we found that the specificity of the SISCOM localisation was poorer for ${ }^{99 \mathrm{~m}} \mathrm{Tc}$ HMPAO studies than for ${ }^{99 \mathrm{~m}} \mathrm{Tc}-\mathrm{ECD}$ studies (table 4). A significantly higher proportion of the ${ }^{99 \mathrm{~m}}$ Tc-HMPAO images in our study were localised to sites that were non-concordant with those localised by intracranial EEG. Also, there was a consistent trend for the ${ }^{99 \mathrm{~m}} \mathrm{Tc}$ HMPAO images to have lower concordance with the localisation by MRI and by ictal scalp EEG, and with the discharge diagnosis. Again it seems that the difference was due to the increased number of postictal injections with ${ }^{99 \mathrm{~m}} \mathrm{Tc}-\mathrm{HMPAO}$, as there was no significant difference between the radioisotopes within the ictal or the postictal subgroups. It is not surprising that injection latency is an important determinant of the sensitivity and the specificity of ictal SPECT. With early ictal studies, the region of hyperperfusion most probably reflects the site of the dominant seizure activity around the time of the radiopharmaceutical injection. ${ }^{69}{ }^{50}$ A late ictal injection allows time for perfusion in secondary areas to become affected by the spread of the seizure activity, and for the activity in the primary epileptogenic zone to wane, resulting in a poorly or a falsely localised study. ${ }^{81} \mathrm{With}$ postictal injections, the situation is even more complicated, as the primary epileptogenic zone often develops prominent postictal hypoperfusion, so that other areas may appear relatively hyperperfused. $^{25253}$ The "positive" subtraction SPECT images of a postictal study may therefore fail to detect hypoperfusion in the primary epileptogenic region, while displaying regions of hyperperfusion in areas that have been secondarily involved by seizure propagation. It is possible that the combined use of "positive" and "negative" subtraction SPECT may improve the sensitivity and specificity of SISCOM when the injection is postictal.

The new stabilised formulation of ${ }^{99 \mathrm{~m}} \mathrm{Tc}$ HMPAO may allow injection latencies to be similar to those we obtained with ${ }^{99 \mathrm{~m}} \mathrm{Tc}$-ECD. None the less, when ictal injections and postictal injections were separately assessed, there was still a consistent trend for the SISCOM images in our ${ }^{99 \mathrm{~m}} \mathrm{Tc}-\mathrm{HMPAO}$ patients to have lower localisation rates, poorer interobserver agreements, and less concordance with the EEG, the MRI, or the discharge diagnosis. This suggests that the timing of injection is not the only factor that explains the difference between the radiopharmaceuticals. Uptake of ${ }^{99 \mathrm{~m}} \mathrm{Tc}$ HMPAO in the background extracerebral tissues has been reported to be higher than that of ${ }^{99 \mathrm{~m}}$ Tc-ECD, possibly due to the slower rate of clearance of ${ }^{99 \mathrm{~m}} \mathrm{Tc}-\mathrm{HMPAO}$ from the blood. ${ }^{24} 54-57$ The results of our quantitative analysis support this finding, with the ${ }^{99 \mathrm{~m}} \mathrm{Tc}-$ HMPAO patients having a significantly lower cortical/extracerebral uptake ratio. The increased extracerebral uptake makes the derivation of a clear and accurate binary image for surface matching more difficult. This difficulty may result in poorer SPECT to SPECT coregistration, which increases the noise in the subtraction images and thereby reduces the sensitivity and specificity of the SISCOM method. Also, some authors have suggested that the grey-white differentiation in uptake may be greater with ${ }^{99 \mathrm{~m}} \mathrm{Tc}-\mathrm{ECD}$ than with ${ }^{99 \mathrm{~m}} \mathrm{Tc}-$ HMPAO SPECT. ${ }^{17}{ }^{54-59}$ The results of our quantitative analyses provide support for this opinion. ${ }^{99 \mathrm{~m}} \mathrm{Tc}-\mathrm{ECD}$ may therefore allow subtle focal changes in cortical uptake to be more readily detected because of their greater contrast against the background activity in the white matter and the extracerebral tissues. ${ }^{54} 55$

Some investigators have suggested that the focal ictal increase in uptake in partial seizures may be less prominent with ${ }^{99 m}$ Tc-ECD than with ${ }^{99 m}$ Tc-HMPAO. ${ }^{19-21}$ Our quantitative analysis of subtraction images disproves the suggestion, as the magnitude of the peri-ictal increase in focal cortical uptake was not significantly different between the radiopharmaceutical groups, for either ictal or postictal injections. Interestingly, the overall magnitude of the relative peri-ictal increase in SPECT uptake did not differ significantly between ictal and postictal studies. The most likely explanation is that postictally, there is a spatially 
progressive blood flow switch, with some regions becoming focally hypoperfused (for example, the lateral temporal neocortex) whereas other regions remain relatively hyperperfused for at least several minutes (for example, the mesial temporal region). ${ }^{12}$ In our study, relatively small ROIs were placed over the region of greatest relative hyperperfusion on the subtraction scan to quantify the maximum peri-ictal increase in radiopharmaceutical uptake. In the post-ictal studies, this ROI was placed over the region with persistent hyperperfusion. Thus, the maximum value for the relative increase in postictal uptake was similar to the value for the ictal scans (although the size of the region of focal hyperperfusion would be expected to be less with postictal studies).

In conclusion our study shows that ${ }^{99 \mathrm{~m}} \mathrm{Tc}-\mathrm{ECD}$ compares favourably with ${ }^{99 \mathrm{~m}} \mathrm{Tc}-\mathrm{HMPAO}$ as a radiopharmaceutical for the performance of peri-ictal SPECT studies of partial seizures. Its use was associated with shorter injection latencies and a higher proportion of ictal injections than with unstablised ${ }^{99 \mathrm{~m}} \mathrm{Tc}-\mathrm{HMPAO}$, thereby enhancing the sensitivity and specificity of localising seizures in intractable partial epilepsy.

1 Newton MR, Berkovic SF, Austin MC, et al. Dystonia, clinical lateralization and regional cerebral blood flow changes in temporal lobe seizures. Neurology 1992;42:371-7.

2 Newton MR, Berkovic SF, Austin MC, et al. Postictal switch in blood flow distribution and temporal lobe seizures. $f$ Neurol Neurosurg Psychiatry 1992;55:891-4.

3 Spencer SS. The relative contributions of MRI, SPECT, and PET imaging in epilepsy. Epilepsia 1994;35(suppl a):S72-89.

4 Mullan BP, O'Connor MK, Hung JC. Single photon emission computed tomography. Neuroimmaging Clin $N \mathrm{Am}$ 1995;5:647-73.

5 Weis $M$, Feistel $H$, Stefan $H$. Utility of ictal SPECT: peri-ictal, post-ictal. Acta Neurol Scand 1994;suppl 152: 145-47.

6 Ho SS, Berkovic SF, Berlangieri SU, et al. Comparison of ictal SPECT and interictal PET in the presurgical evaluation of temporal lobe epilepsy. Ann Neurol 1995;37:738-45.

7 O'Brien TJ, O'Connor MK, Mullan BP, et al. Subtraction ictal SPECT co-registered to MRI in partial epilepsy: description and technical validation of the method with phanto

8 O'Brien TJ, So EL, Mullan BP, et al. Subtraction ictal SPECT co-registered to MRI improves clinical usefulness of SPECT in localizing the surgical seizure focus. Neurology of SPECT in localizi

9 Zubal IG, Spencer SS, Imam K, et al. Difference images calculated from ictal and interictal technetium-99m-HMPAO SPECT scans of epilepsy. F Nucl Med 1995;36:684-9.

10 Weder B, Oettli R, Maguire RP, et al. Partial epileptic seizure with versive movements examined by [ $\left.{ }^{99 m} \mathrm{Tc}\right] \mathrm{HM}-\mathrm{PAO}$ brain single photon emission computed tomography: An early postictal case study analyzed by computerized brain atlas methods. Epilepsia 1996;37:68-75.

11 Chiron C, Jaminska A, Cieuta C, et al. Ictal and interictal subtraction ECD-SPECT in refractory childhood epilepsy. Epilepsia 1997;38(suppl 3):9.

12 Chiron C, Kaminska A, Vera P, et al. Ictal SPECT in infants and children with refractory partial seizures [abstract]. Epilepsia 1997;38 (suppl 8):150.

13 Zubal G, Spanaki-Varelas M, MacMullan J, et al. Quantitative evaluation of postictal SPECT for seizure localization [abstract]. Epilepsia 1997;38(suppl 8):52.

14 Package insert. Ceretec kit for the preparation of technetium $\mathrm{Tc} 99 \mathrm{~m}$ exametazime injection. Arlington Heights, IL, USA: Amersham, 1990.

15 Package Insert. Neurolite kit for the preparation of technetium Tc $99 \mathrm{~m}$ bicisate injection. 1994

16 Grünwald F, Menzel C, Pavics L, et al. Ictal and interictal brain SPECT imaging epilepsy using technetium-99mECD. F Nucl Med 1994;35:1896-901.

17 Lancman ME, Morris HH, Raja S, et al. Usefulness of Ictal and Interictal ${ }^{99 \mathrm{~m}} \mathrm{Tc}$ ethyl cysteinate dimer single photon emission computed tomography in patients with refractory partial epilepsy. Epilepsia 1997;38:466-71.

18 Menzel C, Steidele S, Grünwald F, et al. Evaluation of technitium-99m-ECD in childhood epilepsy. F Nucl Med 1996;37:1106-12.
19 Lee BI, Lee JD, Kim JY, et al. Single photon emission computed tomography-EEG relations in temporal lobe epiputed tomography-EEG relation

20 Kuikka JT, Berkovic SF. Localization of epileptic foci by single-photon emission tomography with new radiotracers. Eur F Nucl Med 1994;21:1173-4.

21 Berkovic SF, Newton MR. Single photon emission tomography. In: Engel J Jr, Pedley TA, eds. Epilepsy: a comprehensive textbook. Philadelphia: Lippincott-Raven, 1997:969-75.

22 Tsuchida T, Nishizawa S, Yanekura Y, et al. SPECT images of technitium-99m-ethyl cysteinate dimer in cerebrovascuar diseases: comparison with other cerebral perfusion tracers and PET. F Nucl Med 1994;35:27-31.

23 Nakagawara J, Nakamura J, Tekeda R, et al. Assessment of postischemic reperfusion and diamox activation test in stroke using ${ }^{99 \mathrm{~m}}$ Tc-EDC SPECT. F Cereb Blood Flow Metab 1994;14:S49-57.

24 Moretti JL, Defer G, Cinotti L, et al. Comparative tomoscintigraphic study of strokes using ECD Tc-99m, HMPAO Tc-99m and IMP I-123, preliminary results [abstract]. Eur f Nucl Med 1988;14:311.

25 Papazyan J-P, Delavelle J, Burkhard P, et al. Discrepancies between HMPAO and ECD SPECT imaging in brain tumors. F Nucl Med 1997;38:592-6.

26 Kuikka JT ME, Vanninen E, Kälviäinen R. Does technitium-99m-bicisate image local brain metabolism in late ictal temporal lobe epilepsy. Eur $\mathcal{f} \mathrm{Nucl}$ Med 1994;21:1247-51.

27 Neirinckx RD, Burke JF, Harrison RC, et al. The retention mechanism of technetium-99m-HMPAO: intracellular reaction with glutathione. F Cereb Blood Flow Metab 1988;37:1694-7.

28 Jacquier-Sarlin MR, Polla BS, Slosman DO. Oxidoreductive state: the major determinant for cellular retention of ${ }^{99 \mathrm{~m}} \mathrm{Tc}$-HMPAO. $\mathcal{F} \mathrm{Nucl}$ Med 1996;34:1367-76.

29 Jacquier-Sarlin MR, Polla BS, Slosman DO. The cellular basis of ECD brain retention. F Nucl Med 1996;37:1694-7.

30 Slosman DO, Jacquier M, Pugin J, et al. Nature of the cellular retention of HMPAO and ECD: an in vitro study [abstract]. F Nucl Med 1995;36:254P.

31 Walovitch RC, Franceschi M, Picard M, et al. Metabolism of 99mTc-L.L-ethyl cysteinate dimer in healthy volunteers. Neuropharmacology 1991;30:283-92.

32 Hung J, Chowdhury S, Redfern M, et al. Rapid preparation method of technetium-99m bicisate. Eur F Nucl Med 1997; 24:655-9.

33 Budde $\mathrm{P}$, Hung J, Mahoney D, et al. Rapid quality control procedure for technetium-99-bicisate. $\mathcal{F}$ Nucl Med Technol 1995;23:190-4.

34 Jiang $\mathrm{H}$, Robb $\mathrm{R}$, Holton $\mathrm{K}$. A new approach to three-dimentional registration of multimodality medical images by surface matching. Visualization in Biomedical Computing 1992;SPIE1808:196-213.

35 Jack CR. Magnetic resonance imaging: neuroimaging and anatomy. Neuroimag Clin N Am 1995;5:597-621.

36 Cohen J. A coefficient of agreement for nominal scales. Educational and Psychological Measurement 1960;20:37-46.

37 Landis JR, Koch G. The measurement of observer agreement for categorical data. Biometrics 1977;33:159-74.

38 Duncan R, Patterson J, Roberts R, et al. Ictal/postictal SPECT in the pre-surgical localization of complex partial seizures. F Neurol Neurosurg Psychiatry 1993;56:141-8.

39 Ho SS, Berkovic SF, McKay WJ, et al. Temporal lobe epilepsy subtypes: differential patterns of cerebral perfusion on ictal SPECT. Epilepsia 1996;37:788-95.

40 Harvey AS, Hopkins IJ, Bowe JM, et al. Frontal lobe epilepsy: clinical seizure characteristics and localization with ictal ${ }^{99 m}$ Tc-HMPAO SPECT. Neurology 1993;43: with ictal

41 Ho SS, Berkovic S, Newton MR, et al. Parietal lobe epilepsy: clinical features and seizure localization by ictal SPECT. Neurology 1994;44:2277-84.

42 Marks DA, Katz A, Hoffer P, et al. Localization of extratemporal epileptic foci during ictal single photon emission computed tomography. Ann Neurol 1992;31:250-5.

43 Newton MR, Berkovic SF, Austin MC, et al. SPECT in the ocalisation of extratemporal and temporal epilepsy. $\mathcal{F} \mathrm{Neu}$ rol Neurosurg Psychiatry 1995;59:26-30.

44 Lee S, Lee S, Bae H, et al. Factors affecting the results of ictal SPECT in medically intractable TLE patients [abstract]. Epilepsia 1996;37(suppl 5):149.

45 Shen W, Lee BI, Park HM, et al. HIPDM-SPECT brain imaging in the presurgical evaluation of patients with intractable seizures. $\mathcal{F}$ Nuc Med 1990;31:1280-4.

46 Berkovic SF, Newton M, Chiron C, et al. Single photon emission tomography. In: Engel J Jr, ed. Surgical treatment of emission tomography. In: Engel J Jr, ed. Surgical trea
the epilepsies. New York: Raven Press, 1993:233-43.

47 Newton MR, Austin MC, Chan JG, et al. Ictal SPECT using technetium-99m-HMPAO: methods for rapid preparation and optimal deployment of tracer during spontaneous seizures. F Nucl Med 1993;34:666-70.

48 Newton MR, Berkovic S, Austin M, et al. Ictal, postictal and interictal single-photon emission tomography in the ateralization of temporal lobe epilepsy. Eur $7 \mathrm{Nucl}$ Med 1994;21:1067-71.

49 Lee BI, Markand ON, Wellman HN, et al. HIPDM-SPECT in patients with medically intractable complex partial seizures. Ictal study. Arch Neurol 1988;45:397-402.

50 Stefan H, Bauer J, Feistel H, et al. Regional cerebral blood flow during focal seizures of temporal and frontocentral onset. Ann Neurol 1990;27:162-6.

51 Jibiki I, Yamaguchi N. Seizure propergation observed from SPECT brain imaging. Fapanese Fournal of Psychiatry and Neurology 1991;45:261-4. 
52 Rowe CC, Berkovic SF, Sia STB, et al. Localization of epileptic foci with postictal single photon emission computed tomography. Ann Neurol 1989;26:660-8.

53 Rowe CC, Berkovic SF, Austin MC, et al. Patterns of postictal cerebral blood flow in temporal lobe epilepsy: quanlitative and quantitative analysis. Neurology 1991;41:109603.

54 Léveillé J, Demonceau G, Walovitch RC. Intrasubject comparison between technitium-99m-ECD and technetium99m-HMPAO in healthy human subjects. $f$ Nucl $\mathrm{Med}$ 1992;33:480-4.

55 Castagnoli A, Borsato $\mathrm{N}$, Bruno A, et al. SPECT brain imaging in chronic stroke and dementia: a comparison of Tc-99m-ECD to Tc-99m-HMPAO. In: Hartmann A, Hoyer S, Kuschinski W, eds. Cerebral ischemia and dementia. Stuttgart: Springer-Verlag, 1991: 327-33.

56 Bruno A, Dosio F, Benti R, et al. Evaluation of a new brain perfusion tracer in humans [abstract]. Eur $\mathcal{f} \mathrm{Nucl}$ Med 1990;16:S187.

57 Saha GB, Macintyre WJ, Go RT. Radiopharmaceuticals for brain imaging. Semin Nucl Med 1994;24:324-49.

58 Friberg L, Andersen AR, Lassen NA, et al. Retention of ${ }^{9{ }_{\mathrm{m}} \mathrm{T}} \mathrm{T}$ c-bicisate as an agent for the measurement of cerebral blood flow with SPECT. I Cereb Blood Flow Metab 1994;14(suppl 1):S19-27.

59 Patterson JC, Early TS, Martin A, et al. SPECT image analysis using statistical parametric mapping: comparison of technetium-99m-HMPAO and technetium-99m-ECD. f Nucl Med 1997;38:1721-5. 